Eur. J. Clin. Chem. Clin. Biochem.

Vol. 32, 1994, pp. 65-69

(ㄷ) 1994 Walter de Gruyter \& Co.

Berlin - New York

\title{
Detection of Tay-Sachs Disease Carriers among Individuals with Thermolabile Hexosaminidase B
}

\author{
By Leah Peleg and B. Goldman \\ Genetic Institute, Sheba Medical Center, Tel-Hashomer, Israel
}

(Received May 17/October 13, 1993)

Summary: The determination of hexosaminidases A and B in most programmes for Tay-Sachs disease carrier detection is based on their different heat sensitivity (hexosaminidase $A$ is the heat labile isoenzyme). This routine cannot be employed for individuals who also possess a thermolabile hexosaminidase B. In Israel, $0.6 \%$ of the screened samples have a labile hexosaminidases B (about 110 each year) and the assessment of their hexosaminidase A activity has hitherto been based on isoenzyme separation by ion exchange chromatography. The latter requires relative large serum samples, and the individuals must usually be reappointed. In order to avoid the thermal treatment we have used the alternative technique, which employs two substrates with different specificities for the two isoenzymes:

1. The fluorogenic substance, 4-methylumbelliferyl-N-acetyl-glucopyranoside, which measures total hexosaminidase activity and

2. the derivative, 4-methylumbelliferyl- $\mathrm{N}$-acetyl glucosamine-6-sulphate, which is considerably more specific toward hexosaminidase A. Hexosaminidase A activity was expressed as a ratio of total activities (the ratio of the assay with 4-methylumbelliferyl-N-acetyl glucosamine-6-sulphate to that with 4-methylumbelliferyl-N-acetyl-glucopyranoside). Using the results from 65 obligate heterozygotes for Tay-Sachs disease, we established our reference ranges for assigning the genotypes with respect to the Tay-Sachs gene. Comparison of the results from 182 unrelated and randomly chosen sera screened by the ratio method and by heat inactivation, showed a very high correlation $(r=0.996)$. Sixty eight sera with thermolabile hexosaminidase B were tested by ion exchange chromatography and by the double substrate method, and they yielded identical diagnoses with regard to the Tay-Sachs locus. The latter strategy showed an improved inter-assay coefficient of variation (11\% instead of $21 \%)$; it also utilizes very small amounts of sera. Results for the estimation of hexosaminidase B heat sensitivity are also presented and analysed.

\section{Introduction}

The basis for the routine estimation of hexosaminidase $\mathrm{A}^{1}$ ) activity, as a means of screening for Tay-Sachs carriers, is the different heat stability of the two main isoenzymes, hexosaminidase $A$ and hexosaminidase $B$. Hexosaminidase $\mathrm{A}$, the heat labile isoenzyme is a heterodimer whose two polypeptide chains $(\alpha$ and $\beta$ ) are

\footnotetext{
1) Enzyme

Hexosaminidase; $N$-acetyl- $\beta-D$-glucosaminidase (EC 3.2.1.52).
}

encoded at two independent loci. Hexosaminidase $A$ is responsible for the degradation of GM2-ganglioside (2acetamido 2-deoxy- $D$-galactopyranose $\beta 1$-4-galactosyl[3-2 $\alpha$-N-acetyl neuraminic acid]- $\beta 1-4$-glucosylceramide) in vivo (1). The absence or impaired activity of hexosaminidase $\mathrm{A}$, due to mutations in the $\alpha$-subunit locus, underlie Tay-Sachs disease. Hexosaminidase B, the thermostable homopolymer, consists of $\beta$-chains only. Previous studies reported pedigrees showing rare mutations in the $\beta$-subunit gene, rendering the hexosaminidase B isoenzyme thermolabile (TLB) (2-4). Hetero- 
zygotes and homozygotes for that trait have been identified and appearcd to be healthy (5). An individual who is a heterozygote for both mutations (thermolabile hexosaminidase B and Tay-Sachs), might be misdiagnosed as a non-carrier of the Tay-Sachs gene. During the thermal treatment, hexosaminidase $A$ and part of hexosaminidase $B$ will be inactivated and the final heat labile fraction might appear normal. Currently, hexosaminidase $\mathrm{A}$ and hexosaminidase $\mathrm{B}$ are separated by ion exchange chromatography and the genotypes are determined according to their final proportions, as previously described $(3,4)$. The latter technique employs a relative large serum sample (5) and the subjects suspected of carrying a labile hexosaminidase B gene are compelled to be reappointed for a second sample. During 19871992, 600 individuals surveyed for the Tay-Sachs gene $(0.59 \%)$ were suspected to be thermolabile hexosaminidase $\mathrm{B}$ carriers (having less than $23 \%$ of serum heat stable activity).

In the present study we used an alternative method, previously described to be appropriate for Tay-Sachs carrier detection, which avoids the heating step (6-8). It employs two different enzymatic assays for each serum sample. Hexosaminidase A activity (measured with the specific substrate, 4-methylumbelliferyl-N-acetyl glucosamine-6-sulphate) is expressed as a fraction of total activity, measured with the common substrate, 4-methylumbelliferyl-N-acetyl-glucopyranoside. We analysed the distribution of hexosaminidase A levels using the latter procedure in 65 obligate Tay-Sachs disease heterozygotes, and were accordingly able to determine the reference ranges for the assignment of the genotypes of the Tay-Sachs allele $(9,10)$. By using the double substrate strategy the first drawn sample was sufficient and the thermolabile hexosaminidase B carriers were absolved from additional tests and anxiety.

\section{Materials and Methods}

The study covers 101690 Jews whose sera were screened for hexosaminidase A level, as part of the national programme for TaySachs disease carrier detection (1987-1992). Serum samples were routinely analysed by an automated system (Technicon autoanalyzer II) which diluted, heat inactivated and assayed hexosaminidase activity (11) using the fluorogenic substrate 4-methylumbelliferyl-N-acetyl-glucopyranoside (Genzyme Fine Chemicals, U.K.). Hexosaminidase A activity was defined as percent of total hexosaminidase activity lost by heating. For ion exchange chromatography, $2-3 \mathrm{ml}$ of dialysed serum were applied to a DE52 (Whatman, U.K.) column $(0.5 \times 5 \mathrm{~cm})$. The effluent contained hexosaminidase $B$, while hexosaminidase $A$ was eluted with 0.3 $\mathrm{mol} / \mathrm{l} \mathrm{NaCl}$ as described $(3,12)$. Each fraction was assayed (13) and the fluorescence was read in a LS-5B Perkin Elmer luminescence spectrometer (U.K.). The overall activity of each isolated isoenzyme was calculated and the genotype was determined according to their final proportions (3). A control serum with a volume identical to the examined sample was used with every run The manual assay with 4-methylumbelliferyl- $\mathrm{N}$-acetyl glucosa- mine-6-sulphate was basically as previously described $(6,7)$ with some modifications as follows: $200 \mu \mathrm{ll}$ of $3 \mathrm{~g} / \mathrm{l}$ 4-methylumbelliferyl-N-acetyl glucosamine-6-sulphate (Research Development Corporation, Canada) in $50 \mathrm{mmol} / \mathrm{l}$ citrate buffer $\mathrm{pH} 4.2$ were added to $100 \mu \mathrm{l}$ aliquots of $1: 10$ diluted serum (in the same buffer) and incubated for $90 \mathrm{~min}$ at $37^{\circ} \mathrm{C}$. The manual assay measuring total activity with 4-methylumbelliferyl- $\mathrm{N}$-acetyl-glucopyranoside (without heating) was carried out at the same time, using the same diluted serum (13). The enzymatic activity with 4-methylumbelliferyl-N-acetyl glucosamine-6-sulphate was expressed either as $\mu \mathrm{mol} / \mathrm{min} \cdot 1$ serum or as the ratio of total activity $(6,7)$. A constant standard was assessed with every run and the activity ratio of each sample was corrected according to the ratio of the standard. In addition a Tay-Sachs patient sample was routinely assayed with the 2 substrates. The limits for the Tay-Sachs genotype assignment were determined according to the range of values and the variance of 65 obligate heterozygotes as previously described $(9,10)$. Hexosaminidase $B$ heat sensitivity was determined with an aliquot of $0.3-0.5 \mathrm{ml}$ of the original serum sample using a $0.5 \times 1 \mathrm{ml}$ DE52 column. The effluent containing the hexosaminidase $B$ fraction $(3,12)$ was heated for $3 \mathrm{~h}$ at $50^{\circ} \mathrm{C}$ after adjustment of the $\mathrm{pH}$ to 5.0 (by addition of about $10 \mu \mathrm{g}$ of citric acid) and the addition of $1 \mathrm{~g} / 1$ bovine serum albumin (13).

\section{Results}

Under our standard conditions, the enzymatic assay with 4-methylumbelliferyl-N-acetyl glucosamine-6-sulphate was linear for more than $2 \mathrm{~h}$ (7), with normal sera and with sera from carriers (data not shown). Serum samples $(n=150)$, previously tested by the routine automated method, were examined by the manual direct assay with 4-methylumbelliferyl-N-acetyl glucosamine-6-sulphate. Figure 1 shows the distribution of hexosaminidase A values, expressed as $\mu \mathrm{mol} / \mathrm{min} \cdot 1$. The mean activity ( $\pm \mathrm{SD}$ ) of $59 \mathrm{normal} \mathrm{sera}$ was $1.49 \pm 0.29 \mu \mathrm{mol} / \mathrm{min} \cdot 1$, the average value of 65 obligate heterozygotes was 0.79 $\pm 0.21 \mu \mathrm{mol} / \mathrm{min} \cdot 1$, and the values of 26 "inconclusive" sera (border line values which could not be designated as carriers or homozygote normals) were scattered between those of normals and heterożygotes. As already reported $(\overline{7}, 8)$, this technique is appropriate for TaySachs carrier screening. In the present conditions there was a significant difference $\left(p<10^{-5}, t\right.$-test) between hexosaminidase A mean values of non-carriers and carriers $(7,8)$. Nevertheless, when individuals bearing the TLB gene were similarly screened (61), they were falsely categorized as carriers (fig. 1), having a mean activity value of $0.95 \pm 0.16 \mu \mathrm{mol} / \mathrm{min} \cdot \mathrm{l}$ (not significantly different from that of the heterozygotes). All the TLB suspected sera were previously diagnosed as noncarriers by the ion exchange chromatography routine.

Entirely different results were obtained when the same hexosaminidase A activity (assayed with 4-methylumbelliferyl-N-acetyl glucosamine-6-sulphate) was expressed as a fraction of total activity, measured with 4methylumbelliferyl-N-acetyl-glucopyranoside (without heating). The distribution of hexosaminidase $A$ values expressed as ratios is shown in figure 2. Hexosaminidase 


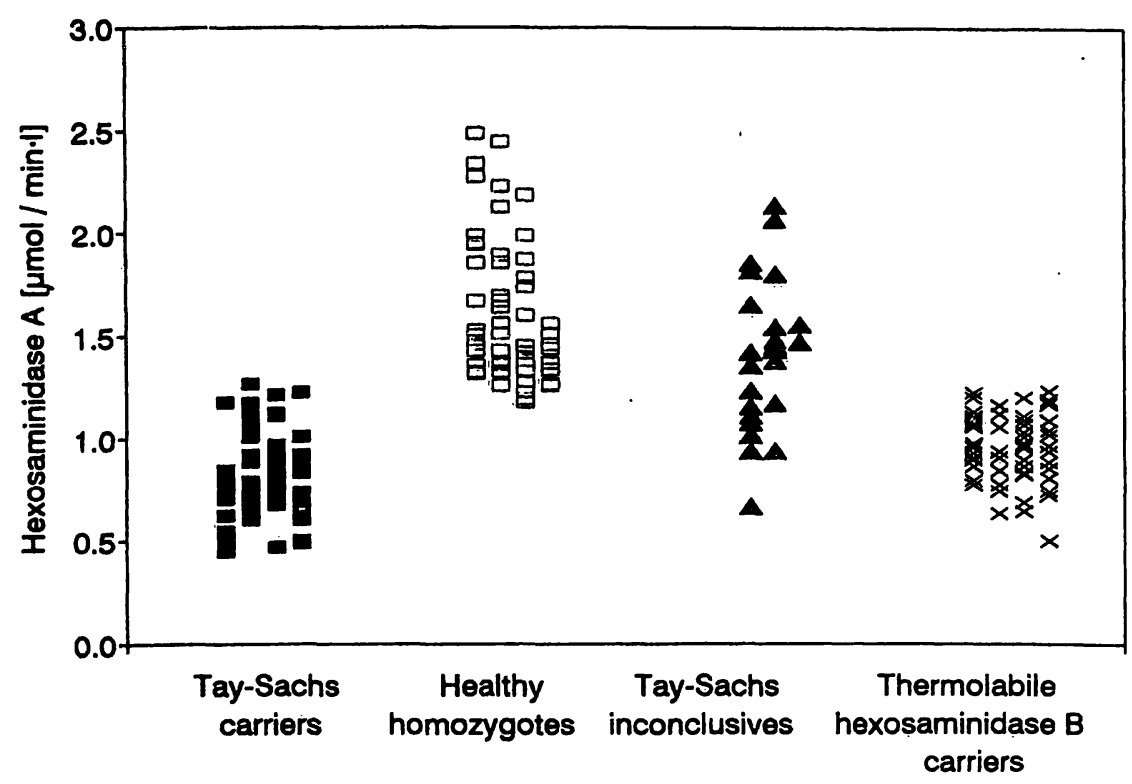

Fig. 1 Hexosaminidase A activity values among the different genotypes of the Tay-Sachs gene. Hexosaminidase A activity was

measured with 4-methylumbelliferyl-N-acetyl glucosamine-6-sulphate and expressed as $\mu \mathrm{mol} / \mathrm{min} \cdot 1$.

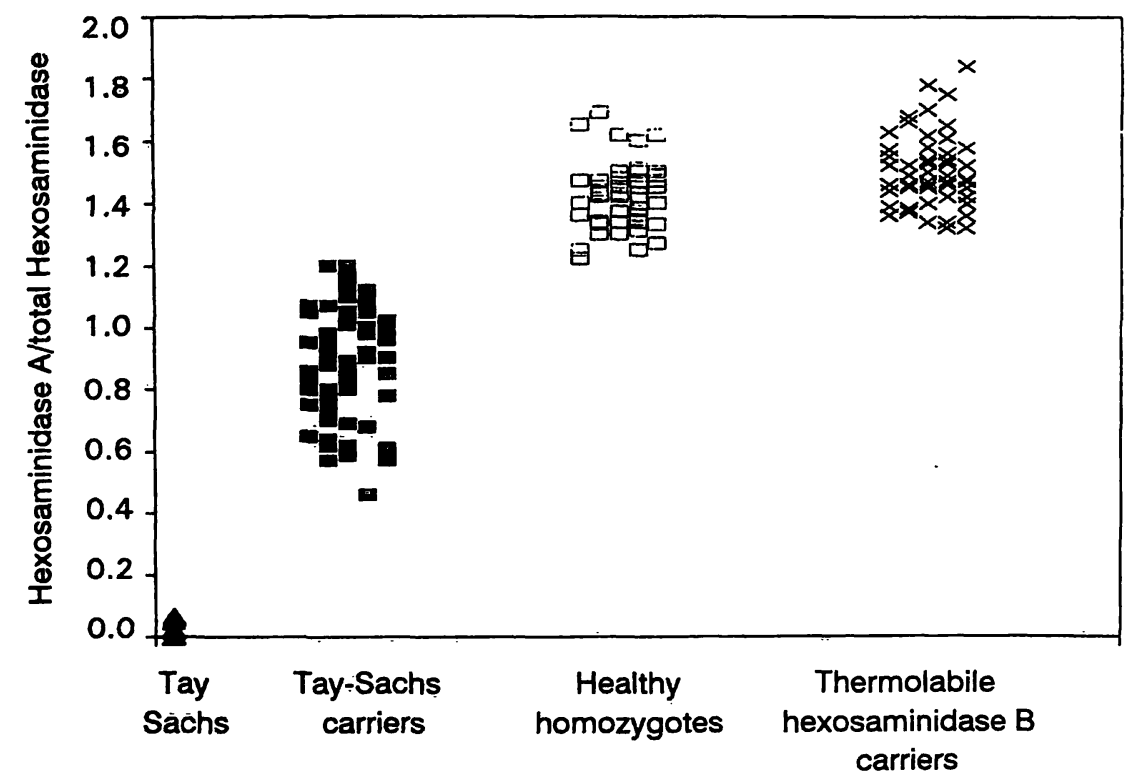

Fig. 2 Hexosaminidase A activity values among different genotypes of the Tay-Sachs gene. Hexosaminidase $\dot{A}$ activity is expressed as ratio of total hexosaminidase activity (4-methylumbelli-
feryl-N-acetyl glucosamine-6-sulphate/4-methylumbelliferyl-Nacetyl-glucopyranoside).
A activities of the TLB-carriers were within the normal range (as expected). The mean ratios of the 3 genotypes were: $1.43 \pm 0.099$ for homozygote normals, 1.48 \pm 0.1 for the 61 TLB individuals and $0.88 \pm 0.17$ for Tay-Sachs obligate heterozygotes, the latter value being significantly different from those of the other 2 groups $\left(p<10^{-6}, t\right.$ test and variance analysis).

The inter-assay coefficient of variation of the double substrate technique (ratio) was $11 \%(\mathrm{~N}=11)$, which is much smaller than that of the ion exchange chromatography procedure $(21 \%, N=12)$. The activity of hexos- aminidase $\mathrm{A}$ as expressed by the ratio mode, exhibited a normal Gaussian distribution (data not shown) similar to the distribution displayed by the conventional heat fractionation method. Thus, we used the same approach to assign the genotypes for the Tay-Sachs locus $(9,10)$. Individuals with a ratio of 1.11 and lower were designated as carriers of the Tay-Sachs allele, those with a value higher than 1.29 being designated as homozygotes for the normal allele, while values in-between were defined as inconclusive. The maximal hexosaminidase A activity found in the sera of Tay-Sachs patients was 0.07 
Tab. 1 Corrclation of the results obtained by the conventional heat inactivation and 4-methylumbelliferyl- $\mathrm{N}$-acetyl glucosamine6-sulphate/4-methylumbelliferyl- $\mathrm{N}$-acetyl-glucopyranoside ratio methods.

\begin{tabular}{rll}
\hline No. of samples & Method & \\
\cline { 2 - 3 } & Conventional & Ratio \\
\hline 168 & Non-carriers & Non-carriers \\
52 & Carriers & Carriers \\
1 & Non-carrier & Inconclusive \\
2 & Inconclusive & Carriers \\
1 & Inconclusive & Non-carrier \\
\hline
\end{tabular}

(15 cases). Based on these limits we found a correlation of $\mathrm{r}=0.9965$ when 182 randomly chosen sera were screened for the Tay-Sachs gene by the two methods, as detailed in table 1.

Diagnoses of the TLB allele obtained by the use of 0.3$0.5 \mathrm{ml}$ of serum were in complete agreement with those obtained by the routine column separation, which employed $2-3 \mathrm{ml}(8)$. Hexosaminidase $\mathrm{B}$ fractions of normal sera eluted from the routine ion exchange column (100 sera) and from the micro column (56 sera), exhibited residual heat stable activity of $91.8 \% \pm 13.6$ and $90.8 \% \pm 5.6$ respectively. Based on the latter averages and tolerance ranges, we designated individuals with more than $20 \%$ heat labile hexosaminidase B as carriers of the TLB gene (very similar to the estimate previously reported, (14)). Eighty sera were diagnosed identically by the two ion exchange procedures and by the hexosaminidase $B$ residual heat stable activity values, with a correlation of $r=0.963$.

\section{Discussion}

About $0.6 \%$ of the individuals attending the Israeli national programme for Tay-Sachs carrier detection are suspected of carrying the TLB gene. Hexosaminidase A activity in those cases was routinely measured by ion exchange chromatography, avoiding the heating step. According to the standard procedure $(5,7)$ the optimal volume of dialysed serum for application to the column was $2-3 \mathrm{ml}$. Smaller samples resulted in large intrabatch variations $(24 \%-35 \%)$ of the final ratio of hexosaminidase A to hexosaminidase B. Since the first drawn sample was not always sufficient for column separation, individuals suspected of carrying the TLB allele (about 600 during 6 years) had to be retested. The modified method, practiced in the present study, employs $0.4-0.5$ $\mathrm{ml}$ serum for the determination of both genotypes. This volume of serum was sufficient for evaluating hexosaminidase $A$ activity and hexosaminidase $B$ heat lability.

It has been reported that total hexosaminidase A activity is consistently lower in the serum of TLB carriers (15). In our laboratory the mean total hexosaminidase activity of 110 sera with TLB as well as their mean hexosaminidase A activity were about $60 \%$ of normal. The decreased hexosaminidase A activity in these individuals stemmed from the overall lower hexosaminidase levels and was not due to a mutation in the $\alpha$ subunit locus. Nevertheless, the original proportions of the main isoenzymes seemed to be sustained, leading to normal activity ratios of hexosaminidase A to total hexosaminidase (fig. 2). The ratio method for detecting hexosaminidase A deficiency is inaccurate when applied to sera from pregnant women, or to the sera of certain inconclusive cases, where the proportions of the various isoenzymes are altered. Several studies report TLB to possess decreased assembly capability and decreased molecular stability $(16,17)$, which probably account for the overall lower measured activity. Since it is not yet clear how many alleles exist for the trait and to what extent hexosaminidase B lability is induced, it is conceivable that some cases with very mild thermolabile hexosaminidase B will be missed by our screening policy. In most reported cases and even in a case of a homozygote for the mutation $(5,16)$, a functional hexosaminidase $A$ can be detected, with sufficient activity to prevent GM2 gangliosidosis. None of the individuals diagnosed through our screening program reported any symptoms when filling out the routine questionnaire before sampling.

The great majority of TLB Jewish carriers originate in Asia (14): Iraq, Iran, Syria, India and Turkey. This mutation was found to be extremely rare among Eastern European Jews '(only one in about 50000), the ethnic groups among which the Tay-Sachs gene is found in substantial frequency. These data are compatible with a previous report which covered the years 1982-1986 (8). During the last few years there has been a consistent increase in the number of non-Ashkenazi and mixed origin Jews attending the screening programme (during 1991 only one third of the screened individuals were Eastern European). Thus the chance of having an individual with both mutations is increasing constantly. It is essential to be alert to any unusual serum sample and if necessary to reassay the sample with 4-methylumbelliferyl-N-acetyl glucosamine-6-sulphate, which provides a fast and reliable determination of hexosaminidase $\mathrm{A}$ activity. 


\section{References}

1. Scriver, C. R., Beaudent, A. L., Sly, W. S. \& Valle, D. (1989) The metabolic basis of inherited diseases. The GM2 gangliosidoses, 6th edn., pp. 1807-1839, McGraw-Hill Company.

2. Hechtman, P. \& Rowlands, A. (1979) Apparent hexosaminidase B deficiency in two healthy members of a pedigree. Am. J. Hum. Genet. 3I, 428-438.

3. Navon, R., Nutman, J., Kopel, R., Gaber, L., Gadoth, N., Goldman, B. \& Nitzan, M. (1981) Hereditary heat-labile hexosaminidase B: Its implication for recognizing Tay-Sachs genotypes. Am. J. Hum. Genet. 33, 907-915.

4. Momoi, T., Kikuchi, K., Shigematsu, Y., Masakatsu, S. \& Taniofa, K. (1983) Prenatal diagnosis of Tay-Sachs disease with heat-labile $\beta$-hexosaminidase B. Clin. Chim. Acta 133, $331-334$.

5. Navon, R., Kopel, R., Nutman, J., Frisch, A., Conzelmann, E., Sandhoff, K. \& Adam, A. (1985) Hereditary heat-labile hexosaminidase B: A variant whose homozygotes synthesize a functional HexA. Am. J. Hum. Genet. 37, 138-146.

6. Inui, K. \& Wenger, D. A. (1984) Usefulness of 4-methylumbelliferyl-6- $\beta$-D-glucopyranoside for the diagnosis of GM2 gangliosidosis in leukocytes. Clin. Genet. 26, 318-321.

7. Ben-Yoseph, Y., Reid, J. E., Shapiro, B. \& Nadler, L. H. (1985) Diagnosis and carrier detection of Tay-Sachs disease: Direct determination of hexosaminidase $A$ using 4-methylumbelliferyl derivatives of $\beta$ - $N$-acetylglucosamine 6 -sulfate and $\beta-N$ acetylgalactosamine-6-sulfate. Am. J. Hum. Genet. 37, 733748.

8. Landels, E. C., Ellis, I. H., Bobrow, M. \& Fenson, A. H. (1991) Tay-Sachs disease heterozygote detection: Use of a centrifugal analyzer for automation of hexosaminidase assays with two different artificial substrates. J. Med. Genet. 28, $101-109$.

9. Kaback, M. M., Shapiro, L. J., Hirsch, P. \& Citra, R. (1977) Tay-Sachs disease heterozygote detection: A Quality control study. In: Progress in Clinical and Biological Research, Alan R. Liśs Inc. N.Y. 18, pp. 267-279.

10. Cantor, R. M., Lim, J. S. T., Roy, C. \& Kaback, M. M. (1985) Sandhoff disease heterozygote detection: A component of population screening for Tay-Sachs disease carriers: Statistical methods. Am. J. Hum. Genet. 37, 912-921.

11. Delvin, E., Pottier, A., Scriver, C. R. \& Gold, R. J. M. (1974) The application of an automated hexosaminidase assay to genetic screening. Clin. Chim. Acta 53, 135-142.

12. Grebner, E. E. \& Jackson, L. G. (1979) Prenatal diagnosis of Tay-Sachs disease: Studies on the reliability of hexosaminidase levels in amniotic fluid. Am. J. Obstet. Genecol. 134, 547550.

13. Padeh, B. \& Navon, R. (1971) Diagnosis of Tay-Sachs disease by hexosaminidase activity in leukocytes and amniotic fluid cells. Israel. J. Med. Sci. 7, 259-263.

14. Navon, R. \& Adam, A. (1990) Thermolabile hexosaminidase (Hex) B: Diverse frequencies among Jewish communities and implication for screening of sera for Hex A deficiencies. Hum. Hered. 40, 99-104.

15. Hechman, P. \& Rowlands, A. (1979) Apparent hexosaminidase $B$ deficiency in two healthy members of a pedigree. Am. J. Hum. Genet. 31, 428-438.

16. Lane, A. B. \& Jenkin, T. (1978) Two variant hexosaminidase $\beta$-chain alleles segregating in a South Africa family. Clin. Chim. Acta 87, 219-228.

17. Dreyfus, J. C., Poenaru, L., Vibert, M., Ravice, N. \& Boue, J. (1977) Characterization of a variant of $\beta$-hexosaminidase: "Hexosaminidase Paris". Am. J. Hum. Genet. 29, 287-293.

Leah Peleg

Genetic Institute

Sheba Medical Center

Tel-Hashomer 52621

Israel 
